\title{
Current Adequacy of the Ophthalmology Workforce in Ontario
}

\section{BACKGROUND}

What is the "adequate" number of ophthalmologists? 1988, National Specialty Physician Review ${ }^{1}$

- 860 ophthalmologists across Canada in 1988.

- Ophthalmologist-to-population ratio was 3.37 per 100,000 population.

- 3.37 ophthalmologists per 100,000 population = "adequate" In 2006, the Ministry of Health and Long-Term Care divided Ontario by geographic location into 14 Local Health Integrated Networks (LHINs).

- LHINs acted as regional health authorities responsible for funding distribution and administration of public healthcare services in the province of Ontario.

In 2019, Ontario introduced the People's Health Care Act. ${ }^{2}$

- Replaces the 14 LHINs with a new entity, Ontario Health.

Population over age 65 bears the majority of sight-threatening conditions, and are the largest sub-population accessing eye care services through an ophthalmologist. ${ }^{3}$

- $56 \%$ of people aged 65 to 74 have evidence of cataract. ${ }^{4}$

- Frequency of eye care utilization during a 12-month period increases with age. ${ }^{3}$

The Canadian population is still aging.

- Baby boomers were born between 1946 and $1965 .^{5}$

- Peak birth was in the year 1960 - there are still 5 years before all baby boomers turn 65 .

\section{OBJECTIVES}

1. To establish the current ophthalmologist-to-population ratio in Ontario and within each geographical region.

2. To determine if ophthalmologist-to-population ratios in Ontario are related to cataract surgery waiting times.

\section{METHODS}

- Cross sectional study.

- Population statistics obtained from 2016 Statistics Canada Census ${ }^{6}$

- A list of all practicing ophthalmologists in Ontario and their main practice location were acquired from CPSO website. ${ }^{7}$

- Each ophthalmologist allocated to a LHIN based on the postal code of his or her primary practice location. ${ }^{8}$

Cataract surgery waiting times were obtained from Health Quality Ontario. ${ }^{9}$

- Waiting time $=$ wait 1 (time to consultation) + wait 2 (time from consultation to cataract surgery)

- Statistical analysis: linear regression in Graphpad Prism 8.

\section{RESULTS}

1. The overall ratio in Ontario is 3.47 ophthalmologists per 100,000 people. However, there is a 5.5-fold range in ratios, from 1.63 in Central West to 9.01 in Toronto Central.

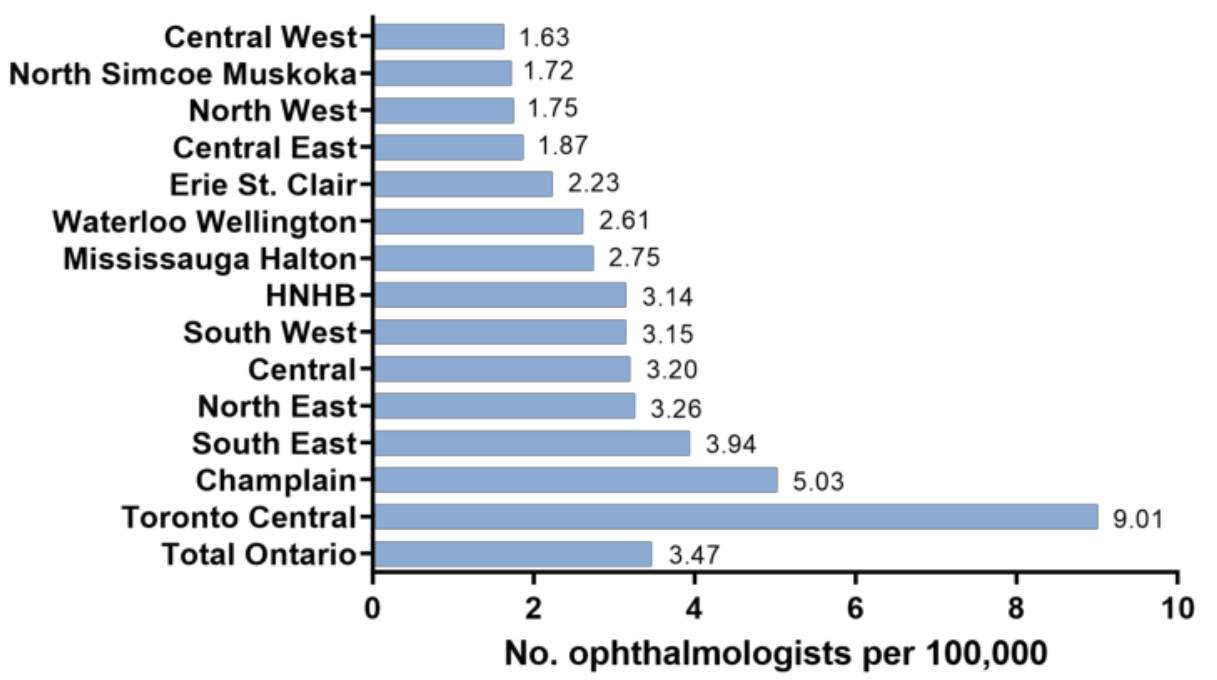

Figure 1. Number of ophthalmologists per 100,000 population across LHINs.

2. As the population above 65 increases, there is a weak correlation to an increas in the number of ophthalmologists per LHIN.

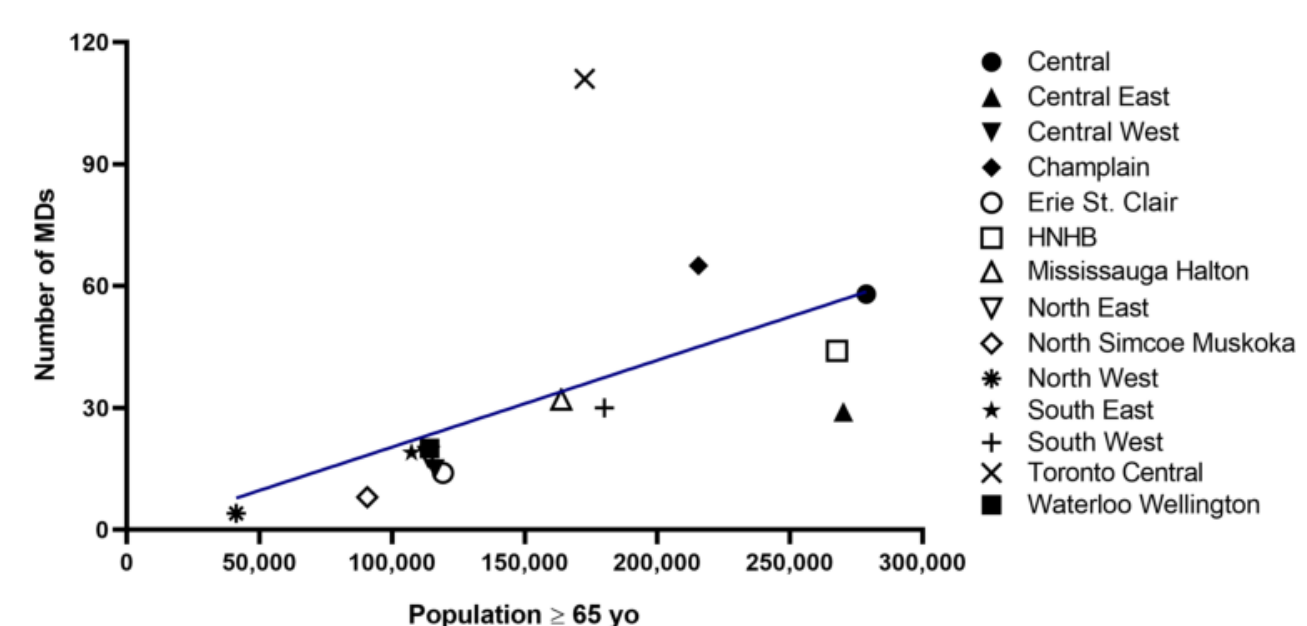

Figure 2. Number of ophthalmologists versus population 65 year and above in each LHIN.

3. The ratio of ophthalmologists per 100,000 people over age 65 ranges from 8.82 in North Simcoe Muskoka to 64.28 ophthalmologists in Toronto Central.

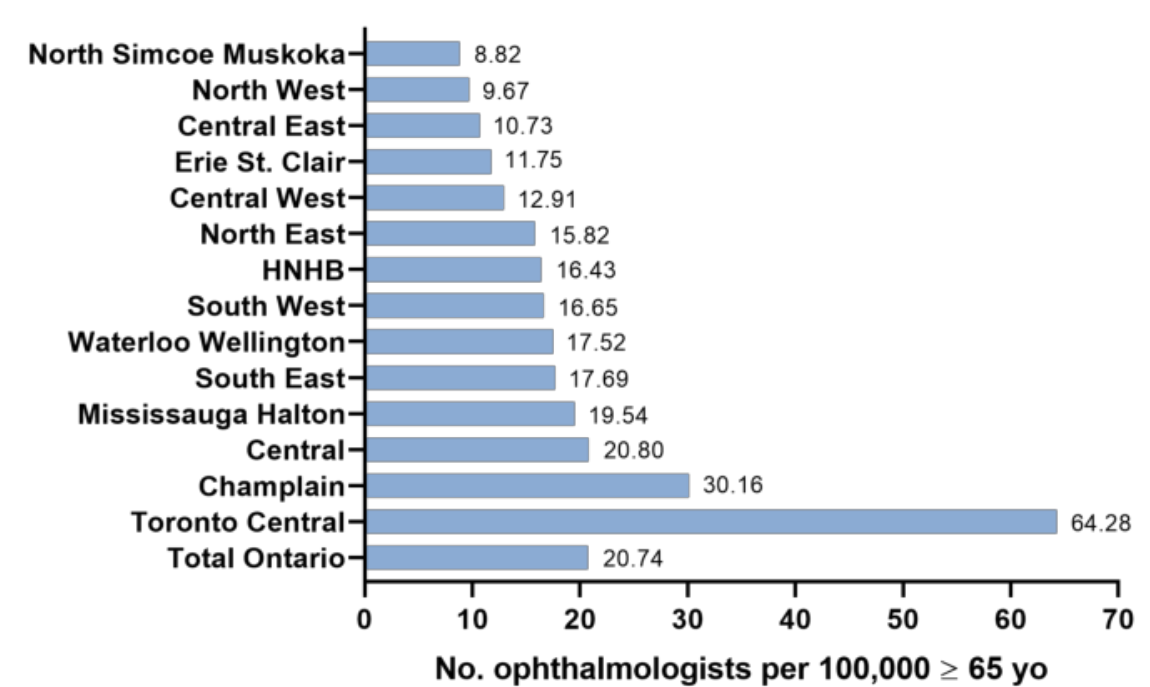

Figure 3. Number of ophthalmologists per 100,000 population aged $65+$ across LHINs.

\section{RESULTS CONT'D}

4. LHINs with a larger age $65+$ population (Figure $4 A$ ) and LHINs with more practicing ophthalmologists (Figure 4B) perform significantly more cataract surgeries per year.
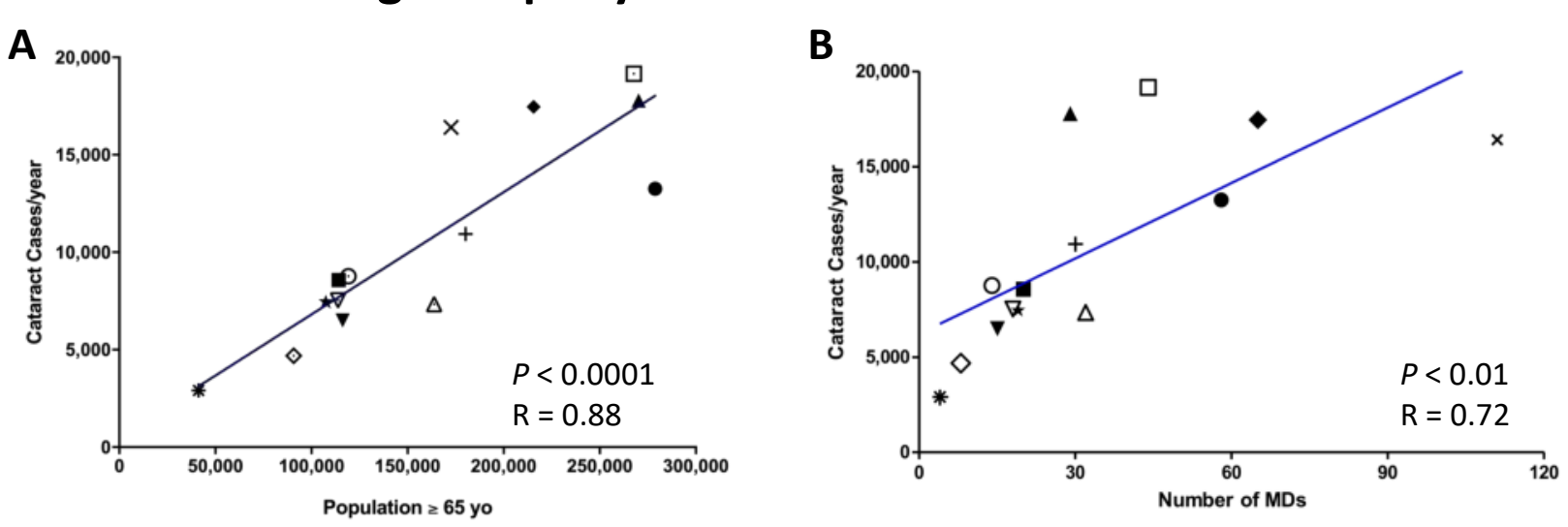

Figure 4. Number of cataract surgeries performed annually versus population aged $65+(A)$ and number of MDs (B) in each LHIN.

5. Areas with a larger number of ophthalmologists did not have a shorter waiting time for cataract surgery.

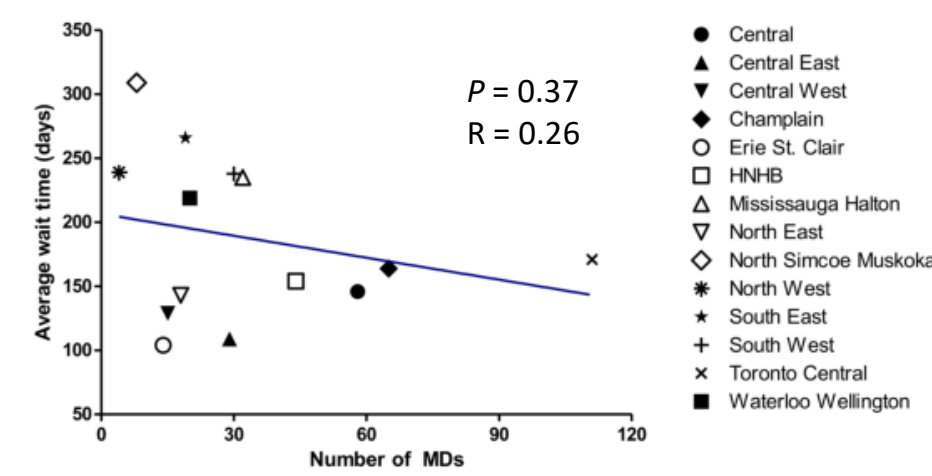

Figure 5. Number of ophthalmologists versus average waiting time for cataract surgery in each LHIN.

\section{DISCUSSION}

Currently the national ratio is keeping pace with the 1988 recommended ratio of 3.37 ophthalmologists to 100,000 people.

- This recommendation did not consider:

- Geographical variation in ophthalmologists.

- Ratio of ophthalmologists to population aged $65+$

Restructuring into Ontario Health allows the government a chance to reassess the adequacy of the ophthalmology workforce in Ontario.

Active recruitment of ophthalmologists to areas with a lower ophthalmologist-to-population ratio, particularly in the 65+ age group, will serve to alleviate the ever-increasing burden of care on existing ophthalmologists in these underserviced areas.

\section{REFERENCES}

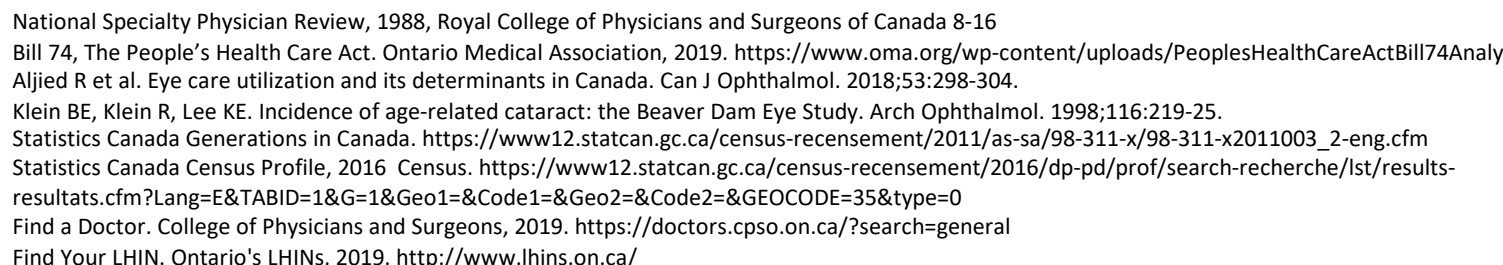

